\title{
Genotypic characterization of human immunodeficiency virus type 1 isolated in Bali, Indonesia in 2016
}

\author{
Siti Qamariyah Khairunisa ${ }^{1 \star}$, Sri Masyeni ${ }^{2 \star}$, Adiana Mutamsari Witaningrum ${ }^{1}$, \\ Muhammad Qushai Yunifiar M. ${ }^{1}$, Dwi Wahyu Indriati ${ }^{1}$, Tomohiro Kotaki ${ }^{3}$, Shuhei Ueda ${ }^{1,3,5}$, \\ Dewa G. Budiyasa ${ }^{4}$, Nasronudin ${ }^{1}$, Masanori Kameoka ${ }^{3,5}$
}

${ }^{1}$ Indonesia-Japan Collaborative Research Centre for Emerging and Re-emerging Infectious Diseases, Institute of Tropical Disease, Airlangga University, Surabaya, Indonesia

${ }^{2}$ Faculty of Medicine, Warmadewa University, Bali, Indonesia

${ }^{3}$ Departemen of International Health, Kobe University Graduate School of Health Science, Hyogo, Japan

${ }^{4}$ Sanjiwani Hospital, Gianyar, Bali, Indonesia

${ }^{5}$ Centre for Infectious Diseases, Kobe University Graduate School of Medicine, Hyogo, Japan

${ }^{*}$ These authors contributed equally to this work

\begin{abstract}
Introduction: Bali is a famous tourism destination in Indonesia, and many international travelers from several foreign countries visit Bali. A large number of human immunodeficiency type 1 (HIV-1) infections was found; however, the genotypes of HIV-1 strains circulating in Bali has not yet been elucidated in detail. In addition, the information on HIV drug resistance (HIVDR) in Bali is limited.

Material and methods: HIV-1 pol gene encoding 31 proteases (PR gene) and 37 reverse transcriptase (RT gene) as well as $47 \mathrm{gag}$ and $33 \mathrm{env}$ genes were successfully amplified from DNA samples extracted from peripheral blood mononuclear cells samples of 51 antiretroviral therapy (ART)-experienced and 7 ART-naïve HIV-1-infected individuals residing in Gianyar and Denpasar, Bali. Genotyping analyses were then performed. The assessment of drug resistance mutations was based on the guidelines published by the International Antiviral Society-United States of America (IAS-USA).

Results: The major HIV-1 subtype detected in this study was CRF01_AE (94.5\%). Recombinant viruses containing CRF01_AE and subtype B genes (5.5\%) were also detected. Drug resistance-associated major mutations were detected in $8 / 31$ (25.8\%) of RT genes but not in PR genes in integrated proviral DNA derived from ART-experienced individuals. However, drug resistance-associated minor mutations were frequently detected in PR genes derived from most samples.

Conclusions: This study revealed that CRF01_AE was the predominant HIV-1 subtype in Bali, Indonesia. In addition, the emergence of drug resistance mutations was evident in proviral DNA derived from ART-experienced individuals, indicating that continuous surveillance is needed in order to monitor the emergence of HIVDR and new viral subtypes in Indonesia.
\end{abstract}

HIV AIDS Rev 2018; 17, 2: 81-90 DOI: https://doi.org/10.5114/hivar.2018.76375

Key words: HIV-1, Bali, Indonesia, subtype, antiretroviral therapy, drug resistance.

Address for correspondence: Dr. Masanori Kameoka, 7-10-2 Tomogaoka, Suma-ku, 654-0142 Kobe, Japan, phone: +81-78-796-4594, e-mail: mkameoka@port.kobe-u.ac.jp
Article history:

Received: 19.07.2017

Received in revised form: 21.11.2017

Accepted: 11.01.2018

Available online: 21.05.2018
International Journal of HIV-Related Problems

HIV \& AIDS

R e v i e w 


\section{Introduction}

Human immunodeficiency virus type-1 (HIV-1) is the major causative agent of acquired immune deficiency syndrome (AIDS) [1]. The virus is characterized by extensive genetic heterogenicity. Due to this variability, HIV-1 is subdivided into 4 groups: $\mathrm{M}$ (major), $\mathrm{O}$ (outlying), $\mathrm{N}$ (new or non-M, non-O), and $\mathrm{P}$ (pending). Viruses in group $\mathrm{M}$, which are responsible for the majority of infections in the worldwide HIV-1 pandemic [2,3], are further classified into many subtypes or circulating recombinant forms (CRFs). Of these, subtypes A, B, C, D, and G, as well as CRF01_AE and CRF02_AG are the major subtypes and CRFs responsible for the worldwide pandemic. Intersubtype genetic variability has been reported among subtypes and CRFs; $15-20 \%$ of genetic variability is found in the gag gene and $25-35 \%$ in the env gene [1].

While subtype B of HIV-1 is the predominant subtype in the Americas, Europe, and Australia [4, 5], and is also present as a minority in Asia [6], a growing epidemic of non-B subtypes and CRFs has been reported in Africa [7] and Asia [8]. CRF01_AE is prevalent throughout Southeast Asia and responsible for more than $90 \%$ of infection cases in Indonesia [9]. Furthermore, several recombinant forms between CRF01_AE and subtype B, including CRF33_01B, have emerged in Indonesia [9-13]. Different subtypes and CRFs are considered to show different rates of disease progression, immune responses, responses to antiretroviral therapy (ART), and the development of drug resistance [14]. Therefore, it is important to monitor the global prevalence of subtypes and CRFs for the prevention and control of HIV-1 as well as vaccine development.

Antiretroviral therapy (ART) has markedly reduced the transmission, morbidity, and mortality associated with HIV. In 2013, number of HIV-infected individuals in Indonesia with $\mathrm{CD} 4{ }^{+} \mathrm{T}$-cell count less than 500 cells $/ \mathrm{mm}^{3}$ were estimated to be 510,000 , and these individuals were eligible for ART according to the World Health Organization (WHO) criteria. The Indonesian national ART program enabled $40 \%$ of these individuals to access ART in 2011 [15]. The first-line regimen of ART recommended in Indonesia is a combination of two nucleoside reverse-transcriptase inhibitors (NRTIs) and a non-nucleoside reverse-transcriptase inhibitor (NNRTI). Lamivudine (3TC), zidovudine (AZT), tenofovir (TDF), nevirapine (NVP), and efavirenz (EFV) are commonly used. In patients with virological failure and/or adverse effects to the first-line regimen, ritonavir-boosted protease inhibitors in combination with two NRTIs are recommended as the second-line regimen. Other drugs, including didanosine (ddI), etravirine (ETR), and rilpivirine (RPV), are uncommon in the country. Although ART is successful in Indonesia, the emergence of drug resistance has been reported among treatment failure cases as well as ARTnaïve individuals [16-19].

Bali is a famous tourism destination in Indonesia, and many international travelers from several foreign countries visit Bali. It is assumed that some of these travelers may be HIV-infected. According to Indonesian Ministry of Health, Bali has the fourth largest number of HIV cases in Indonesia.
Previous studies revealed a large number of HIV infection in Bali $[20,21]$. However, the genotypes of HIV-1 strains circulating in Bali has not yet been elucidated in detail. In addition, the information on HIVDR in Bali is limited. In order to clarify the circulating HIV-1 subtypes and monitor the emergence of HIVDR in Bali, a genotypic study was conducted on viral genomic fragments derived from peripheral blood samples of ART-experienced or ART-naïve HIV-1infected individuals residing in Gianyar and Denpasar, Bali.

\section{Material and methods}

\section{Ethics statement}

This study was conducted with approvals from the Medical Research Ethics Committees of the Faculty of Medicine of Udayana University, Airlangga University, and Kobe University Graduate School of Medicine. All study participants were enrolled after providing written informed consents.

\section{Study site, patient recruitment, and sample collection}

Fifty-eight HIV-infected individuals, consisting of 51 ART-experienced and 7 ART-naïve individuals, were recruited from the Sinta HIV Clinic of Sanjiwani Hospital in Gianyar regency and from Puskesmas Denpasar Selatan II at Denpasar municipality in Bali, Indonesia. Eight milliliters whole blood samples were collected from study participants between January and March 2016. Peripheral blood mononuclear cells (PBMC) were isolated by density gradient centrifugation for 10 minutes at 1,800 rpm using the BD Vacutainer ${ }^{\circ} \mathrm{CPT}^{\mathrm{m} \mathrm{m}}$ Cell Preparation Tube (Becton, Dickinson and Company, Franklin Lakes, NJ, USA). DNA was then extracted from PBMCs using the QIAamp DNA Blood Mini Kit (Qiagen, Hilden, Germany). Demographic, clinical, and hematological data as well as disease severity according to the WHO guidelines of study participants were retrieved from the medical records.

\section{HIV-1 genotypic analysis}

The HIV-1 pol gene encoding protease (PR gene) and reverse transcriptase (RT gene) as well as gag and env genes were amplified by nested polymerase chain reaction (PCR) using Gotaq green master mix (Promega, Madison, WI, USA). The primer sets used were as follows. In order to amplify the viral PR gene, the primers DRPRO5, 5'-AGACAGGYTAATTTTTTAGGGA-3' ((corresponding to nucleotides (nt) 2074-2095 of an HIV-1 reference strain, HXB2 (GenBank accession no. K03455)) and DRPRO2L, 5' -TATGGATTTTCAGGCCCAATTTTTGA-3' (nt 2716 to 2691) were used for the first PCR, and the primers DRPRO1M, 5'-AGAGCCAACAGCCCCACCAG-3' (nt 2148 to 2167), and DRPRO6, 5'-ACTTTTGGGCCATCCATTCC-3' (nt 2611 to 2592) were used for nested PCR. In order to amplify the viral RT gene, RT1L, 5' -ATGATAGGGGGAATTGGAGGTTT-3' (nt 2388 
to 2410) and DRRT4L, 5'-TACTTCTGTTAGTGCTTTGGTTCC-3' (nt 4402 to 4380) were used for first PCR, and RT7L, 5'-GACCTACACCTGTCAACATAATTGG-3' (nt 2485 to 2509) and DRRT6L, 5' -TAATCCCTGCATAAATCTGACTTGC-3' (nt 4309 to 4285) were used for nested PCR. In order to amplify the gag gene encoding Gag p24, the primers for first PCR were H1G777, 5'-TCACCTAGAACTTTGAATGCATGGG-3' (nt 1231 to 1255) and H1P202, 5'-CTAATACTGTATCATCTGCTCCTGT-3' (nt 2352 to 2325), while the primers used for nested PCR were H1Gag1584, 5'AAAGATGGATAATCCTGGG-3' (nt 1577 to 1595) and G17, 5'-TCCACATTTCCAACAGCCCTTTTT-3' (nt 2040 to 2017). In order to amplify the $\mathrm{C} 2-\mathrm{V} 3$ regions of env gene, the primers used for first PCR were M5, 5'-CCAATTCCCATACATTATTGTGCCCCAGCTGG-3' (nt 6858 to 6889) and M10, 5'-CCAATTGTCCCTCATATCTCCTCCTCCAGG-3' (nt 7661 to 7632), while the primers used for nested PCR were M3, 5'-GTCAGCACAGTACAATGIACACATGG-3' (nt 6948 to 6973) and M8, 5'-TCCTTGGATGGGAGGGGCATACATTGC-3' (nt 7547 to 7521). The PCR product amplified at the end-point dilution of DNA templates were subjected to a sequencing analysis in order to examine the genomic fragment of the major viral population in a sample.

The BigDye Terminator version 3.1 Cycle Sequencing kit and ABI PRISM3500Xl genetic analyzer (Applied Biosystems, Foster City, CA, USA) were used in the sequencing analysis of amplified HIV-1 gag, pol, and env genes. Data were assembled using Genetyx version 10 software (Genetyx, Tokyo, Japan). HIV-1 subtyping was conducted using the Recombinant Identification Program (RIP) available on the HIV sequence database website (www.hiv.lanl.gov) and jumping profile Hidden Markov Model (jpHMM)-HIV (http:// jphmm.gobics.de/submission_hiv). Neighbor-joining trees with a Kimura two-parameter model were constructed using MEGA6.2 software with bootstrap values (1,000 replicates) for relevant nodes being reported on a representative tree. When a discrepancy was found in the subtyping of a sample among the gag, PR, RT, and env genes, it was defined as a unique recombinant form of more than two HIV-1 subtypes and CRFs. The appearance of drug resistant mutations in the $31 \mathrm{PR}$ and $37 \mathrm{RT}$ genes was studied according to the International Antiviral Society-United States of America (IAS-USA) panel [22]. The nucleotide sequences of the PR, RT, gag, and env genes have been registered in the GenBank database under accession numbers KY985644-KY985676 (env genes), KY985677-KY985723 (gag genes), KY985724KY985760 (RT genes), and KY985761-KY985791 (PR genes).

\section{Results}

\section{Demographic and clinical information of study participants}

The demographic information of 58 study participants is shown in Table 1 . The mean age of study participants was 36.5 (SD \pm 11.66 ) years old. Study participants had a median CD4 cell count of 52.50 cells $/ \mathrm{mm}^{3}$, with an interquartile range
Table 1. Demographic characteristics of study participants

\begin{tabular}{|c|c|}
\hline Characteristics & Value $(n=58)$ \\
\hline Age, mean, years (SD) & $36.54(11.66)$ \\
\hline CD4, pre-ART, median (IQR) & $52.50(1-480)$ \\
\hline \multicolumn{2}{|l|}{ Gender, $n(\%)$} \\
\hline Male & $37(63.8 \%)$ \\
\hline Female & $21(36.2 \%)$ \\
\hline \multicolumn{2}{|l|}{ Marital status, $n(\%)$} \\
\hline Married & $48(82.8 \%)$ \\
\hline Single (divorced/widowed) & $10(17.2 \%)$ \\
\hline \multicolumn{2}{|l|}{ Ethnic group, $n$ (\%) } \\
\hline Bali & $55(94.8 \%)$ \\
\hline Java & $2(3.5 \%)$ \\
\hline East Nusa Tenggara & $1(1.7 \%)$ \\
\hline \multicolumn{2}{|l|}{ Transmission risk category, $n$ (\%) } \\
\hline Heterosexual & $51(87.9 \%)$ \\
\hline Injecting drug use & $1(1.7 \%)$ \\
\hline Men who have sex with men & $4(6.9 \%)$ \\
\hline Mother-to-child transmission & $2(3.4 \%)$ \\
\hline \multicolumn{2}{|l|}{ Types of ART used, $n(\%)$} \\
\hline $\mathrm{AZT}+3 \mathrm{TC}+\mathrm{NVP}$ & $10(17.3 \%)$ \\
\hline $\mathrm{AZT}+3 \mathrm{TC}+\mathrm{EFV}$ & $6(10.3 \%)$ \\
\hline $\mathrm{TDF}+3 \mathrm{TC}+\mathrm{EFV}$ & $26(44.8 \%)$ \\
\hline $\mathrm{TDF}+3 \mathrm{TC}+\mathrm{NVP}$ & $10(17.3 \%)$ \\
\hline Naïve & $6(10.3 \%)$ \\
\hline \multicolumn{2}{|l|}{ Duration of ART, $n(\%)$} \\
\hline$<1$ year & $15(25.9 \%)$ \\
\hline $1-3$ years & $23(39.7 \%)$ \\
\hline$>3$ years & $14(24.1 \%)$ \\
\hline \multicolumn{2}{|l|}{ HIV stage } \\
\hline Non-AIDS & $14(24.1 \%)$ \\
\hline AIDS & $44(75.9 \%)$ \\
\hline \multicolumn{2}{|l|}{ History of OI, $n$ (\%) } \\
\hline No & $7(12.1 \%)$ \\
\hline Yes & $51(87.9 \%)$ \\
\hline
\end{tabular}

(IQR) of 1-480 cells $/ \mathrm{mm}^{3}$. Most ethnic group were from Bali (94.8\%). The main route of infection was heterosexual intercourse (87.9\%). Six individuals were ART-naïve (10.3\%), while 52 individuals had been on ART (89.7\%). The fixed combination of AZT/3TC/NVP (17.3\%), AZT/3TC/EFV (10.3\%), TDF/3TC/EFV (44.8\%), or TDF/3TC/NVP (17.3\%) was used in the treatment of individuals on ART. The mean duration of ART usage was $26.68(\mathrm{SD} \pm 17.84)$ months. Fourteen individuals were in the non-AIDS stage (24.1\%), while 44 individuals were in the AIDS stage (75.9\%). Fifty-one individuals had developed opportunistic infections (87.9\%). 
Table 2. HIV-1 subtyping

\begin{tabular}{|c|c|c|c|c|c|}
\hline \multirow{2}{*}{$\begin{array}{l}\text { Sample } \\
\text { ID }\end{array}$} & \multirow{2}{*}{$\begin{array}{c}\text { Subtype/ } \\
\text { CRF assignment }\end{array}$} & \multicolumn{4}{|c|}{ Subtyping } \\
\hline & & PR gene & RT gene & gag gene & env gene \\
\hline H01 & CRF01_AE & $\mathrm{NA}^{*}$ & CRF01_AE & CRF01_AE & NA \\
\hline $\mathrm{HO2}$ & CRF01_AE & NA & CRF01_AE & NA & NA \\
\hline $\mathrm{H} 03$ & CRF01_AE & NA & NA & CRF01_AE & CRF01_AE \\
\hline $\mathrm{H} 04$ & NA & NA & NA & NA & NA \\
\hline H05 & CRF01_AE & NA & NA & CRF01_AE & NA \\
\hline H06 & CRF01_AE & CRF01_AE & CRF01_AE & CRF01_AE & NA \\
\hline H07 & CRF01_AE & NA & CRF01_AE & CRF01_AE & NA \\
\hline $\mathrm{H} 08$ & CRF01_AE & CRF01_AE & NA & CRF01_AE & CRF01_AE \\
\hline H09 & NA & NA & NA & NA & NA \\
\hline $\mathrm{H} 010$ & CRF01_AE & NA & CRF01_AE & CRF01_AE & CRF01_AE \\
\hline H011 & CRF01_AE/B* & NA & NA & CRF01_AE & B \\
\hline H012 & NA & NA & NA & NA & NA \\
\hline $\mathrm{H} 013$ & CRF01_AE & NA & NA & CRF01_AE & NA \\
\hline H014 & CRF01_AE & CRF01_AE & NA & CRF01_AE & NA \\
\hline H015 & CRF01_AE & NA & CRF01_AE & CRF01_AE & CRF01_AE \\
\hline $\mathrm{H} 016$ & CRF01_AE & CRF01_AE & CRF01_AE & CRF01_AE & CRF01_AE \\
\hline $\mathrm{H} 017$ & CRF01_AE & NA & NA & CRF01_AE & NA \\
\hline H018 & CRF01_AE & CRF01_AE & CRF01_AE & CRF01_AE & NA \\
\hline H019 & CRF01_AE & NA & CRF01_AE & CRF01_AE & CRF01_AE \\
\hline $\mathrm{H} 020$ & CRF01_AE & CRF01_AE & NA & NA & CRF01_AE \\
\hline $\mathrm{H} 021$ & CRF01_AE & NA & NA & CRF01_AE & NA \\
\hline $\mathrm{H} 022$ & CRF01_AE & NA & CRF01_AE & NA & NA \\
\hline $\mathrm{H} 023$ & CRF01_AE & CRF01_AE & CRF01_AE & NA & CRF01_AE \\
\hline $\mathrm{H} 024$ & CRF01_AE & CRF01_AE & NA & CRF01_AE & CRF01_AE \\
\hline $\mathrm{H} 025$ & CRF01_AE & NA & CRF01_AE & CRF01_AE & NA \\
\hline H026 & CRF01_AE & NA & CRF01_AE & CRF01_AE & CRF01_AE \\
\hline $\mathrm{H} 027$ & CRF01_AE & CRF01_AE & CRF01_AE & CRF01_AE & NA \\
\hline $\mathrm{H} 028$ & CRF01_AE & CRF01_AE & NA & NA & NA \\
\hline H029 & CRF01_AE & NA & CRF01_AE & CRF01_AE & CRF01_AE \\
\hline $\mathrm{H} 030$ & CRF01_AE/B & NA & CRF01_AE & B & CRF01_AE \\
\hline H031 & CRF01_AE & NA & NA & CRF01_AE & CRF01_AE \\
\hline H032 & CRF01_AE & NA & CRF01_AE & CRF01_AE & CRF01_AE \\
\hline $\mathrm{H} 033$ & CRF01_AE & NA & CRF01_AE & CRF01_AE & CRF01_AE \\
\hline $\mathrm{H} 034$ & CRF01_AE & NA & NA & CRF01_AE & CRF01_AE \\
\hline $\mathrm{H} 035$ & CRF01_AE & CRF01_AE & CRF01_AE & CRF01_AE & CRF01_AE \\
\hline $\mathrm{H} 036$ & CRF01_AE & CRF01_AE & CRF01_AE & CRF01_AE & CRF01_AE \\
\hline $\mathrm{H} 037$ & CRF01_AE & NA & CRF01_AE & CRF01_AE & CRF01_AE \\
\hline $\mathrm{H} 038$ & CRF01_AE & NA & CRF01_AE & NA & NA \\
\hline H039 & CRF01_AE & NA & CRF01_AE & CRF01_AE & CRF01_AE \\
\hline $\mathrm{H} 040$ & CRF01_AE & CRF01_AE & NA & CRF01_AE & CRF01_AE \\
\hline $\mathrm{H} 041$ & CRF01_AE & CRF01_AE & CRF01_AE & CRF01_AE & CRF01_AE \\
\hline $\mathrm{H} 042$ & CRF01_AE & CRF01_AE & CRF01_AE & NA & NA \\
\hline H043 & CRF01_AE & CRF01_AE & CRF01_AE & CRF01_AE & CRF01_AE \\
\hline
\end{tabular}


Table 2. Cont.

\begin{tabular}{l|c|c|c|c|c}
\hline \multirow{2}{*}{$\begin{array}{l}\text { Sample } \\
\text { ID }\end{array}$} & $\begin{array}{c}\text { Subtype/ } \\
\text { CRF assignment }\end{array}$ & PR gene & RT gene & gag gene & env gene \\
\cline { 3 - 5 } & CRF01_AE & CRF01_AE & CRF01_AE & CRF01_AE & CRF01_AE \\
\hline $\mathrm{H} 045$ & CRF01_AE & CRF01_AE & CRF01_AE & CRF01_AE & CRF01_AE \\
\hline $\mathrm{H} 046$ & CRF01_AE & CRF01_AE & CRF01_AE & CRF01_AE & NA \\
\hline H047 & CRF01_AE & CRF01_AE & CRF01_AE & CRF01_AE & CRF01_AE \\
\hline H048 & CRF01_AE & CRF01_AE & NA & NA & NA \\
\hline H049 & CRF01_AE & CRF01_AE & NA & CRF01_AE & CRF01_AE \\
\hline H050 & CRF01_AE & CRF01_AE & CRF01_AE & CRF01_AE & CRF01_AE \\
\hline H052 & CRF01_AE/B & B & CRF01_AE & CRF01_AE & NA \\
\hline H053 & CRF01_AE & CRF01_AE & CRF01_AE & CRF01_AE & NA \\
\hline H054 & CRF01_AE & CRF01_AE & NA & CRF01_AE & CRF01_AE \\
\hline H055 & CRF01_AE & CRF01_AE & CRF01_AE & CRF01_AE & CRF01_AE \\
\hline H056 & CRF01_AE & CRF01_AE & CRF01_AE & CRF01_AE & CRF01_AE \\
\hline H057 & CRF01_AE & CRF01_AE & CRF01_AE & CRF01_AE & NA \\
\hline H058 & CRF01_AE & CRF01_AE & CRF01_AE & CRF01_AE & CRF01_AE \\
\hline
\end{tabular}

${ }^{*}$ Not available due to the failure of $P C R$

**Recombinant form of HIV-1 containing viral gene fragments of CRFO1_AE and subtype $B$

Table 3. Distribution of HIV-1 subtypes by gender

\begin{tabular}{l|c|c|c}
\hline \multirow{2}{*}{ HIV-1 subtype } & \multicolumn{3}{|c}{ HIV/AIDS patients } \\
\cline { 2 - 4 } & Total, $\boldsymbol{N}=55$ & Male, $n=35$ & Female, $n=20$ \\
\hline CRF01_AE & $52(94.5 \%)$ & $34(97.1 \%)$ & $18(90 \%)$ \\
\hline CRF01_AE/B* & $3(5.5 \%)$ & $1(2.9 \%)$ & $2(10 \%)$ \\
\hline
\end{tabular}

${ }^{*}$ Recombinant viruses between CRFO1_AE and subtype $B$

\section{HIV-1 subtyping}

The sequencing data of $31 \mathrm{PR}$ genes (297 bp; nt 2253 to 2,549), 37 RT genes (762-bp; nt 2,550 to 3,311), the partial fragments of 47 gag genes encoding Gag p24 (382-bp; nt 1,627 to 2,008), and the partial fragments of $33 \mathrm{env}$ genes spanning the $\mathrm{C} 2-\mathrm{V} 3$ region (389-bp; nt 7,020 to 7,408) were obtained from 55 blood samples, while no sequencing data was available from 3 samples (Table 2). According to RIP and jpHMMHIV results as well as the phylogenetic tree analysis, CRF01_ $\mathrm{AE}$ was the dominant subtype in Bali (94.5\%) (Table 3). In addition, recombinant viruses containing CRF01_AE and subtype B gene fragments (5.5\%) were detected (Table 3). Viral subtyping by RIP, jpHMM-HIV, and phylogenetic trees showed consistent results (data not shown).

\section{Appearance of drug resistance- associated mutations}

The appearance of drug resistance-associated mutations was evaluated on successfully sequenced 31 RT and 26 PR genes derived from ART-experienced individuals. Drug resistance-associated major mutations against NRTIs and NNRTIs were found in $8 / 31$ RT genes (25.8\%) in integrated proviral DNA derived from ART-experienced individuals (Table 4). In particular, samples H033 and H056 contained several major mutations, including thymidine analogue-associated mutations (TAMs), which confer multi NRTI resistance (Table 4). In PR genes, no drug resistance-associated major mutations were detected (data not shown). However, minor mutations, M36I ((amino acid substitution from methionin (M) to isoleucine (I) at position 36 in PR gene)) (100\%), H69K (100\%), and L89M (88.5\%) were commonly detected in the PR gene (Table 4) [21]. These mutations confer resistance to ritonavir-boosted indinavir (IDV/r), atazanavir (ATV/r), fosamprenavir (FPV/r), lopinavir (LPV/r), tipranavir (TPV/r), and nelfinavir (NFV).

\section{Discussion}

HIV-1 genetic diversity is caused by viral mechanisms that elude immune control and drug pressure. The existence of distinct subtypes and CRFs reflects the presence of a dy- 
Table 4. Antiretroviral drug resistance-associated mutations in 8 individuals on ART

\begin{tabular}{|c|c|c|c|c|}
\hline \multirow[t]{2}{*}{ Sample ID } & \multirow[t]{2}{*}{ ART regimen and duration } & \multicolumn{3}{|c|}{ Drug resistance mutations* } \\
\hline & & NRTI & NNRTI & PI \\
\hline H010 & $\begin{array}{c}\text { FDC, } \\
\text { more than } 3 \text { years }\end{array}$ & M184V & $\begin{array}{l}\text { Y181C } \\
\text { Y188L }\end{array}$ & $N A^{* *}$ \\
\hline H018 & $\begin{array}{c}\text { FDC, } \\
2 \text { years }\end{array}$ & $-* * *$ & E138G & $\begin{array}{c}\text { M36I } \\
\text { H69K } \\
\text { V82I } \\
\text { L89M } \\
193 \mathrm{~L}\end{array}$ \\
\hline $\mathrm{H} 030$ & FDC, 18 months & - & V90I & NA \\
\hline H033 & $\begin{array}{c}3 \mathrm{TC}+\mathrm{AZT}+\mathrm{EFV} \\
43 \text { months }\end{array}$ & $\begin{array}{c}\text { M41L } \\
\text { D67N } \\
\text { K70R } \\
\text { M184V } \\
\text { L210W } \\
\text { T215Y } \\
\text { K219E }\end{array}$ & $\begin{array}{c}\text { A98G } \\
\text { K103N } \\
\text { V108I }\end{array}$ & NA \\
\hline $\mathrm{H} 035$ & EFV TRIPLE, 2 years & M184V & $\begin{array}{l}\text { K103N } \\
\text { Y188L }\end{array}$ & $\begin{array}{l}\text { G16E } \\
\text { K20R } \\
\text { M36I } \\
\text { H69K } \\
\text { L89M } \\
\end{array}$ \\
\hline $\mathrm{H} 043$ & FDC & M184V & K103N & $\begin{array}{l}\text { G16E } \\
\text { K20R } \\
\text { M36I } \\
\text { H69K } \\
\text { L89M }\end{array}$ \\
\hline H055 & $\begin{array}{c}3 \mathrm{TC}+\mathrm{AZT}+\mathrm{NVP} \\
18 \text { months }\end{array}$ & - & M30L & $\begin{array}{l}\text { K20R } \\
\text { M36I } \\
\text { H69K } \\
\text { V77I } \\
\text { L89M }\end{array}$ \\
\hline H056 & $\begin{array}{c}\text { TDF+3TC+EFV } \\
11 \text { months }\end{array}$ & $\begin{array}{c}\text { D67N } \\
\text { K70R } \\
\text { K219E }\end{array}$ & Y181C & $\begin{array}{l}\text { G16E } \\
\text { M36I } \\
\text { H69K } \\
\text { L89M }\end{array}$ \\
\hline
\end{tabular}

*The assessment of drug resistance mutations was based on the guidelines published by the International Antiviral Society-United States of America (IAS-USA). Drug resistance-associated major mutations are shown in bold

${ }^{* *}$ Not available due to the failure of $P C R$

${ }^{* * *}$ Not detected

namic genetic evolutionary process of the virus. The most prominent HIV-1 subtype in Bali found in the present study was CRF01_AE, which is widely prevalent throughout Southeast Asian countries including Indonesia [8, 9, 17]. Previous HIV-1 genotyping studies in Indonesia reported similar findings. Roselinda and Jekti [23] found that CRF01_AE viruses circulated in 7 provinces in Indonesia as the predominant subtype, followed by subtype B and recombinant viruses containing subtypes A and G gene fragments, which were not found in the present study. CRF01_AE was found in $80 \%$ of the samples in North Sumatra province and in East Java, while it was only detected in a quarter of samples in the Papua provinces $[23,24]$. A study in Malaysia, a neighbor coun- try of Indonesia, reported similar findings for the HIV-1 subtype distribution in that CRF01_AE was the most dominant strain and other subtypes were identified as minorities [25]. In contrast to this study, subtype B (41.9\%) was the major subtype prevalent in Sorong, West Papua, Indonesia [11]. Differences in the distribution of the HIV-1 subtype in Indonesia may be caused by many factors such as different high-risk behaviors for HIV-1 infection, genetic and demographic characteristic of individuals residing in each region, geographic, economic, and social factors. Diverse high-risk behaviors sustain HIV-1 transmission in different regions of the world, and multiple transmission routes may be involved in spreading the HIV-1 epidemic within the same 
A

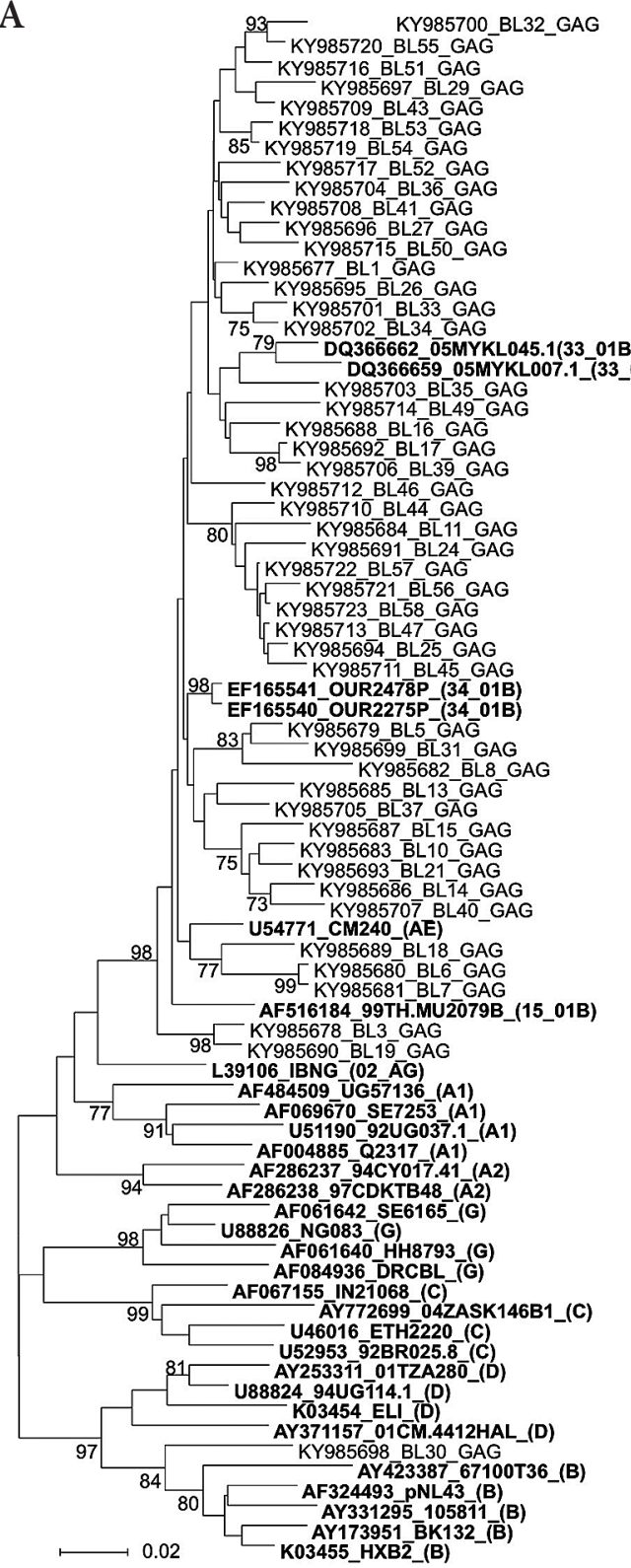

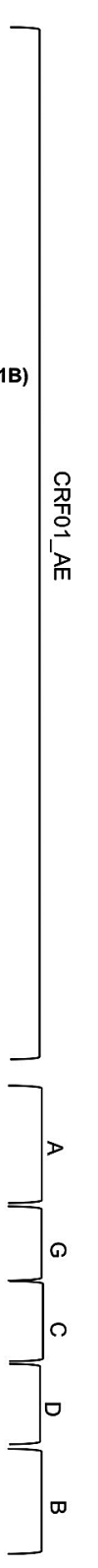

B

87 KY985774_BL41_PR KY985775_BL42_PR

KY985786 B

$75-K Y 985787$ BL54_PR Y985785_BL52_PR

KY985788_BL55_PR

KY985769_BL27_PR

KY985783 BL50 PR

KY985776_BL43_PR

KY985771_BL35_PR

KY985782_BL

KY985779_BL46 PR

KY985766_BL20_PR

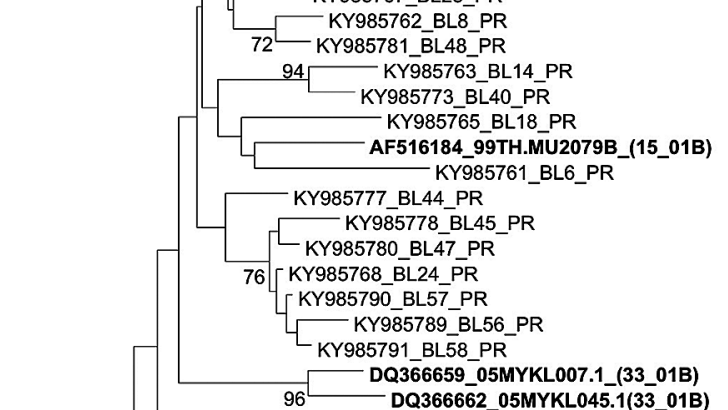

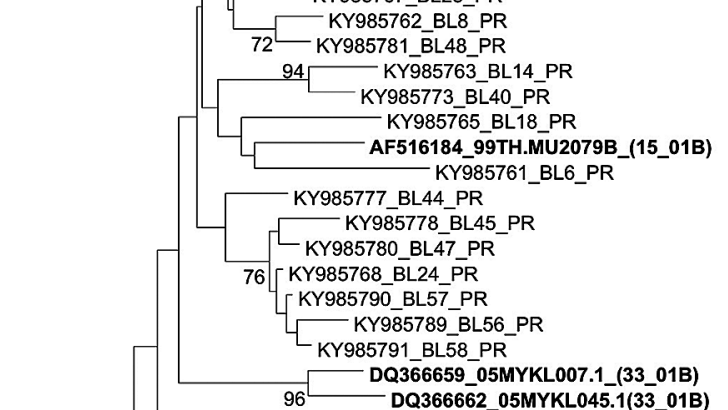

96 DQ366662_05MYKL045.1(33_01B) AF484509_UG57136_(A1)

U51190_92UG037.1_(A1)

AF004885_Q2317_(A1)

AF069670_SE7253_(A1)

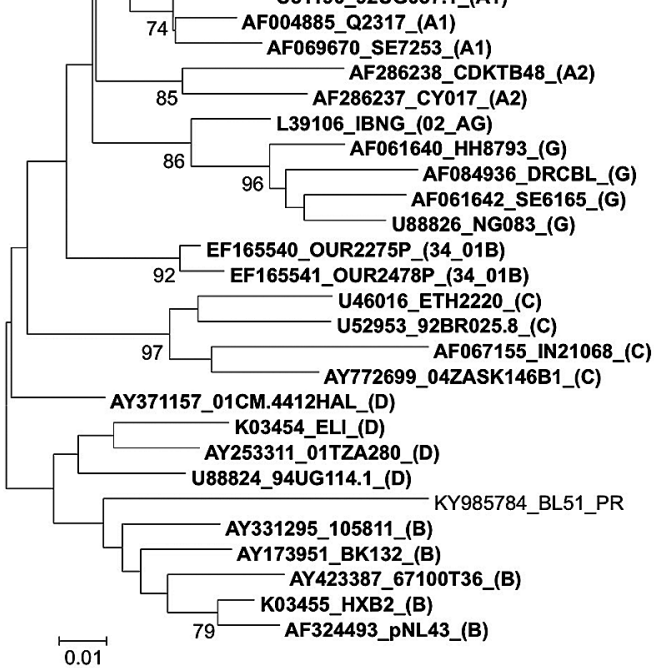

0.01

Fig. 1. Phylogenetic analysis of HIV-1 PR, RT, gag, and env gene sequences. Phylogenetic trees were generated for the newly sequenced HIV-1 gag (A), PR (B), RT (C), and env genes (D) together with the corresponding viral genes of reference HIV-1 strains representing subtype $A 1(A 1)$, subtype $A 2(A 2)$, subtype $B(B)$, subtype $C(C)$, subtype $D(D)$, subtype $G(G)$, CRF01_ AE (01_AE), CRF02_AG (02_AG), CRF15_01B (15_01B), CRF33_01B (33_01B), and CRF34_01B (34_01B). The reference strains of the HIV-1 subtype are shown in bold. Sequence codes are presented as the GenBank accession number, patient ID or name of the reference strain, and the subtype or CRF of the reference strain (shown in parentheses) in order. Bootstrap values were shown when values were $>70$. CRF - circulating recombinant form

region [1]. As an RNA virus, HIV-1 replication is characterized by high mutation rates, leading to diversification of its strains. Intersubtype recombination contributes to HIV-1 sequence diversity, which is generated by the co-circulation of two or more different subtypes and/or CRFs in high-risk populations [26]. Other recombinant forms, such as recombinant viruses between CRF01_AE and subtype B (5.5\%) were also detected in the present study. The prevalence of recombinant forms of HIV-1 in Indonesia was reported to be low. Namely, the low prevalence of recombinants between CRF01_AE and subtype B was similar to previous findings in Surabaya (3.0\%) and in Sorong $(2.3 \%)[11,17]$. Subtype B is the most prevalent HIV-1 subtype in North America, Western Europe, and Australia; however, Sides et al. found that non-B subtypes 
C

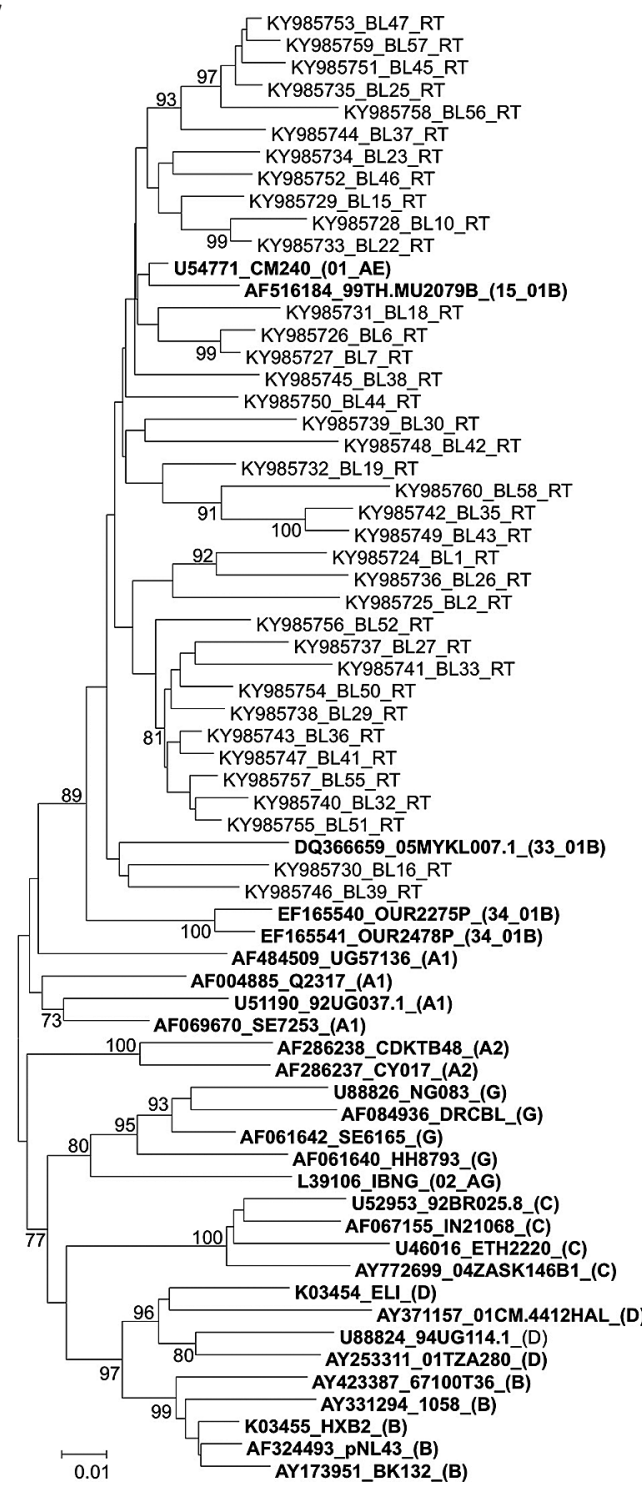

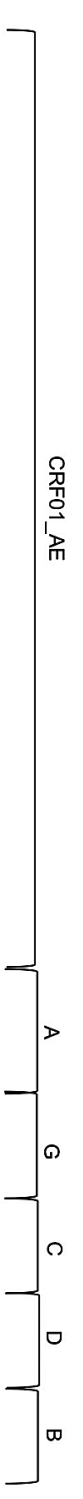

$\mathrm{D}$

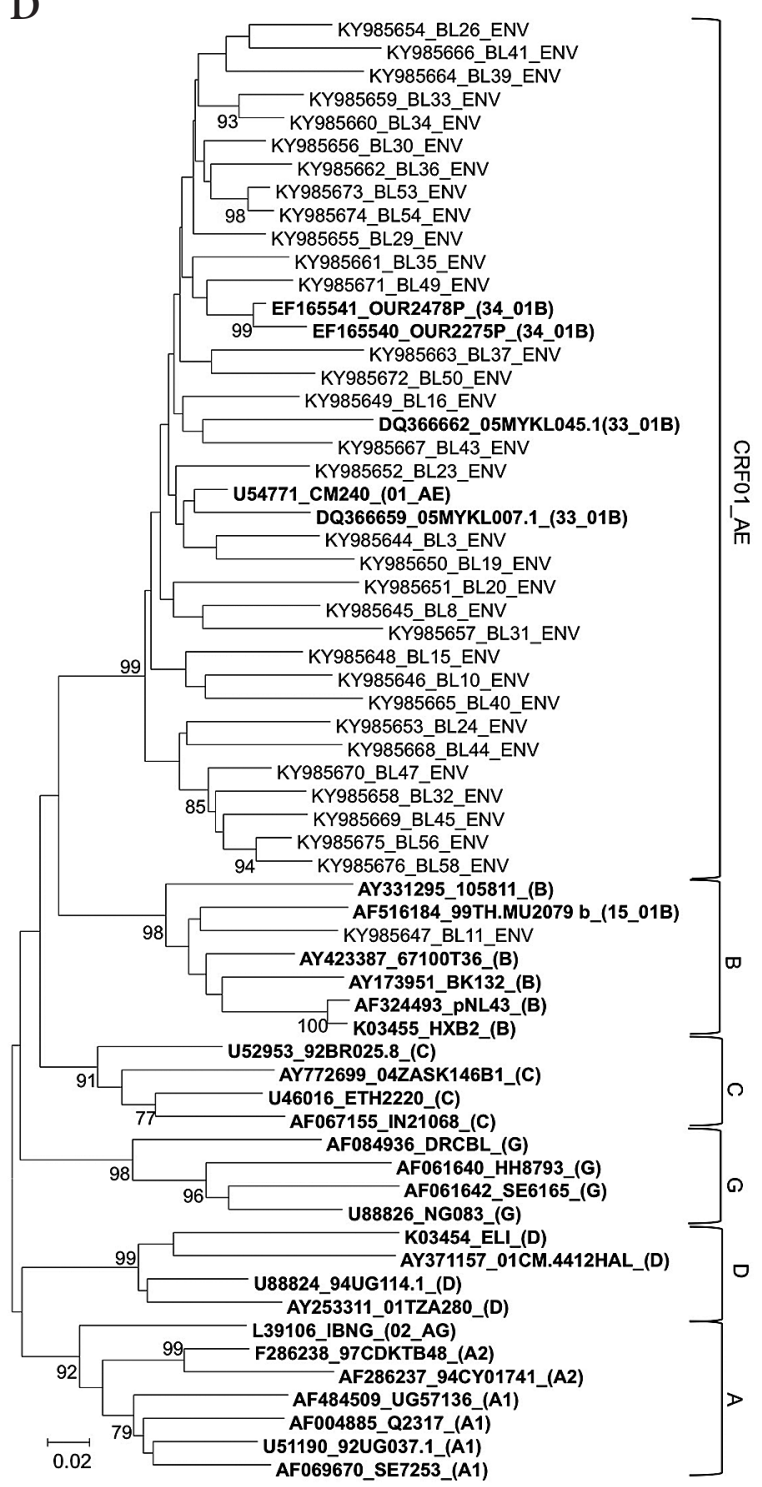

Fig. 1. Cont.

were prevalent as the major subtypes among African-born HIV-1-infected individuals in Minnesota, USA [27], suggesting that migration may have affected the subtype distribution in the world. Furthermore, the epidemic of a minor lineage of HIV-1 subtype B (termed subtype B') occurred in Asia [6]. Therefore, it is important to continuously monitor the prevalence of HIV-1 subtypes and CRFs worldwide.

Genetic variability was found in viral PR and RT genes coding the viral enzymes targeted by antiretroviral drugs. If a drug resistance-associated mutation appears as a result of a genetic polymorphism, it may be selected further by drug-selective pressure, and markedly influence therapeutic outcome [1]. In the present study, drug resistance-associated major mutations were detected in RT genes derived from 8 ART-experienced individuals. In particular, several muta- tions were detected in 2 individuals treated with the combination of 2 NRTIs and 1 NNRTI. Patient H033 had been treated with 3TC/AZT/EFV for 43 months. Several TAMs (M41L, D67N, K70R, and M184V) and mutations against NNRTIs (K103N, V108I) was found in the viral RT gene derived from the patient. Based on clinical data, this patient had not developed any opportunistic infections, ART side effect, or ART failure (data not shown). The mutations, M184V and $\mathrm{K} 103 \mathrm{~N}$ are commonly detected as multi-drug resistance mutations leading to the virological failure of a first-line NRTIs- and NNRTI-based regimens $[16,28]$. The appearance pattern of these drug resistance mutations was similar with those in previous studies in Indonesia, Malaysia, Taiwan, Turkey, and USA [17, 18-31]. M184V is a common mutation in treated populations; however, it is very rare among 
ART-naive individuals. This may be due to that M184V deteriorates viral fitness in the absence of ART. In present study, drug resistance-associated major mutations were not found in RT genes derived from ART-naïve patients, while several TAMs (M41L, D67N, K70R, L210W, T215Y, K219E) were detected in ART-naïve individuals as transmitted drug resistance (TDR) in a previous study conducted in Surabaya, Indonesia [19]. Due to a limited sample size, the emergence of TDR among ART-naïve individuals cannot be evaluated in this study. Since drug resistance-associated mutations have frequently been detected among treatment-failure patients in Indonesia, there may be a risk of TDR among newly infected individuals. This study needs to be continued with a larger sample size, improved sampling quality, and direct sequencing in order to obtain the best TDR estimation in HIV-1-infected individuals in Indonesia. In addition, several minor mutations were found in PR genes derived from most study participants. Although these mutations may also affect clinical manifestations, this was not evaluated in present study. Minor mutations have been detected in PR genes of non-B subtypes, including CRF01_AE, as natural polymorphisms [14]. Drug resistance-associated minor mutations in PR genes have also been reported in other studies in Indonesia $[11,17,18]$.

\section{Conclusions}

CRF01_AE is the dominant HIV-1 strain prevalent in Bali. The emergence of drug resistance-associated major mutations was evident among patients on ART. The detection of drug resistance-associated mutations in ART-naïve and ART experienced samples, and the combining of data with clinical, immunological, and virological data of HIV-1-infected individuals are needed in order to improve the clinical outcomes of ART in Indonesia.

\section{Acknowledgements}

MK and N conceived and supervised the study; SQK and TK designed experiments; SQK, AMW, MQYM, and DWI performed experiments; SM, DHB, and AC collected samples and clinical information; AQK, SM, AMW, MQYM, DWI, TK, and SU analyzed data; SQK and SM wrote the manuscript; SU and MK made manuscript revisions. This work was supported by the Japan Initiative for the Global Research Network on Infectious Diseases (J-GRID) from the Ministry of Education, Culture, Sport, Science, and Technology in Japan, and the Japan Agency for Medical Research and Development (AMED); and the Institute of Tropical Disease as the Center of Excellence (COE) program by the Ministry for Research and Technology (RISTEK) of Indonesia. The funders had no role in study design, data collection, and analysis, the decision to publish, or preparation of the manuscript. The manuscript was proofread by Medical English Service, Kyoto, Japan.

\section{Conflict of interest}

The authors declare no potential conflicts of interest with respect to the research, authorship, and/or publication of this article.

\section{References}

1. Buonaguro L, Tornesello ML, Buonaguro FM. Human immunodeficiency virus type 1 subtype distribution in the worldwide epidemic: pathogenetic and therapeutic implications. J Virol 2007; 81: 10209-10219.

2. Van HF, Li Y, Neel C, et al. Human immunodeficiency viruses: SIV infection in wild gorilla. Nature 2006; 444: 164.

3. Sharp PM, Bailes E, Chaudhuri RR, et al. The origin of acquired immune deficiency syndrome viruses: where and when? Philos Trans R Soc Lond B Biol Sci 2001; 356: 867-876.

4. Leitner T, Korber B, Daniels M, et al. HIV-1 subtype and circulating recombinant form (CRF) reference sequences, 2005. Access on 8 June 2017. Available at: https://www.hiv.lanl.gov/content/sequence/HIV/COMPENDIUM/2005/RefSeqs05.pdf

5. Abecasis AB, Wensing AM, Paraskevis D, et al. HIV-1 subtype distribution And its demographic determinants in newly diagnosed patients in Europe suggest highly compartmentalized epidemics. Retrovirology 2013; 10: 7.

6. Li Y, Uenishi R, Hase S, et al. Explosive HIV-1 subtype B' epidemics in Asia driven by geographic and risk group founder events. Virology 2010; 402: 223-227.

7. Wilkinson E, Engelbrecht S, de Oliveira T. History and origin of the HIV-1 subtype C epidemic in South Africa and the greater south African region. Scientific Report 2015; 5: 16897.

8. Hemelaar J, Gouws E, Ghys PD, Osmanov S. Global and regional distribution of HIV-1 genetic subtypes and recombinants in 2004 . AIDS 2006; 20: W13-23.

9. Kotaki T, Khairunisa SQ, Sukartiningrum SD, et al. High prevalence of HIV-1 CRF01_AE viruses among female commercial sex workers residing in Surabaya, Indonesia. PLoS One 2013; 8: e82645.

10. Oyomopito RA, Chen YJ, Sungkanuparph S, et al. Risk group characteristics and viral transmission cluster in South-East Asian patients infected with HIV-1 circulating recombinant form (CRF) 01_AE and subtype B. Kaohsiung J Med Sci 2015; 31: 445-453.

11. Witaningrum AM, Kotaki T, Khairunisa SQ, et al. Genotypic characterization of human immunodeficiency virus type 1 derived from antiretroviral therapy-naive individuals residing in Sorong, West Papua. AIDS Res Hum Retroviruses 2016; 32: 812-817.

12. Sahbandar IN, Takahashi K, Djoerban Z, et al. Current HIV type 1 molecular epidemiology profile and identification of unique recombinant forms in Jakarta, Indonesia. AIDS Res Hum Retroviruses 2009; 25: 637-646.

13. Sahbandar IN, Takahashi K, Motomura K, et al. The Indonesian Variants of CRF33_01B: Near-Full Length Sequence Analysis. AIDS Res Hum Retroviruses 2011; 27: 97-102.

14. Taylor BS, Sobieszczyk ME, McCutchan FE, Hammer SM. The challenge of HIV-1 subtype diversity. N Engl J Med 2008; 358: 15901602 .

15. Indonesian National AIDS Commission. Republic of Indonesia Country Report on the follow up to the Declaration of Commitment on HIV/AIDS (UNGASS), Reporting Period 2010-2011. Accessed on 9 June 2017. Available at http://files.unaids.org/en/dataanalysis/ knowyourresponse/countryprogressreports/2012countries/ce ID_Narrative_Report.pdf

16. Fibriani A, Wisaksana R, Indriati A, et al. Virological failure and drug resistance during first line anti-retroviral treatment in Indonesia. J Med Virol 2013; 85: 1394-1401. 
17. Khairunisa SQ, Kotaki T, Witaningrum AM, et al. Appearance of drug resistance-associated mutations in human immunodeficiency virus type 1 protease and reverse transcriptase derived from drug-treated Indonesian patients. AIDS Res Hum Retroviruses 2013; 31: 255-259.

18. Kotaki T, Khairunisa SQ, Sukartiningrum SD, et al. Detection of drug resistance-associated mutations in human immunodeficiency virus type 1 integrase derived from drug-naive individuals in Surabaya, Indonesia. AIDS Res Hum Retroviruses 2014; 30: 489-492.

19. Kotaki T, Khairunisa SQ, Witaningrum AM, et al. HIV-1 transmitted drug resistance mutations among antiretroviral therapy-naive individuals in Surabaya, Indonesia. AIDS Res Ther 2015; 12: 5.

20. Adnyani S, Sawitri AAS, Wulandari L, et al. Factors associated with zidovudine substitution in HIV/AIDS patients attending Badung Hospital, Bali, Indonesia between 2006-2014. Intern J Res Med Sci 2016; 4: 5028-5033.

21. Masyeni S, Utama S, Somia A, Merati TP. Factors influencing bone mineral density in ARV-naive HIV infected patients at Sanglah Hospital, Bali. Acta Med Indones 2013; 45: 175-179.

22. Wensing AM, Calvez V, Gunthard HF, et al. 2017 update of drug resistance in HIV-1 infection. Top Antivir Med 2017; 24: 132-133.

23. Roselinda, Jekti RP. Genotype and transmission of human immunodeficiency virus-1 in seven provinces in Indonesia. Health Sci Indones 2012; 3: 27-31.

24. Ismail YS, Soetjipto S, Wasito EB, Nasronudin. HIV genotype analysis from HIV infected patients in East Java area. Journal Natural 2012; 12: 23-29.

25. Chook JB, Ong LY, Takebe Y, et al. Molecular detection of HIV-1 subtype B, CRF01_AE, CRF33_01B and newly emerging recombinant lineages in Malaysia. Am J Trop Med Hyg 2015; 92: 507-512.

26. Malim MH, Emerman M. HIV-1 sequence variation: drift, shift, and attenuation. Cell 2001; 104: 469-472.

27. Sides TL, Akinsete O, Henry K, et al. HIV-1 subtype diversity in Minnesota. J Infect Dis 2005; 192: 37-45.

28. Derache A, Shin H, Balamane M, et al. HIV drug resistance mutations in proviral DNA from a community treatment program. PLoS One 2015; 10: e0117430.

29. Mohamad S, Deris ZZ, Yusof NK, et al. Assessing subtypes and drug resistance mutations among HIV-1 infected children who failed antiretroviral therapy in Kelantan, Malaysia. Braz J Infect Dis 2012; 16: 284-288.

30. Weng YW, Tsai H, Lee SS, et al. Prevalence and associated factors for HIV-1 transmitted drug resistance in voluntary clients for counseling and testing in Southern Taiwan. J Microbiol Immunol Infect 2016; 49: 487-493.

31. Celen MK, Sayan M, Dal T, et al. Molecular epidemiology of HIV-1 strains in the south-east and east of Turkey. Asian Pac J Trop Biomed 2015; 5: 773-777. 